\title{
GEODESICS ON SURFACES OF REVOLUTION
}

\author{
BY \\ DONALD P. LING
}

Introduction. We are concerned in this paper with the behavior in the large of the geodesic lines on a class of surfaces of revolution. The central theme is the number and the distribution of the double points of these geodesics, and one of the main theorems establishes a "zoning" of the surfaces in a manner dictated by this distribution. A second theorem sets up a classification of admissible surfaces on the basis of the number of the double points of its geodesic lines.

An admissible surface $S$ is formed by revolving about $O y$ a curve which rises monotonically from the origin to infinity as $x$ increases, and which possesses a continuously turning tangent (save possibly at certain exceptional points). On every geodesic of $S$ there is a point $P$, the "point of symmetry," which is nearest to the vertex of $S$, and at which the curve is tangent to a parallel of $S$. The two branches of the geodesic proceed in either direction from $P$, and spiral symmetrically in opposite senses around the axis of $S$ toward infinity. Under special conditions the two branches may fail to intersect one another. More generally, however, they cut each other repeatedly in a sequence of double points, which may conveniently be numbered by starting with the one nearest the vertex. We may thus speak of the "first double point," the "second double point," and so on. This sequence may be finite or infinite, but if it is finite for one geodesic of $S$ it is finite for all. The above discussion is of course not meant to apply to the meridians of $S$, whose special nature is perfectly clear.

A discrete sequence of parallels, $P_{1}, P_{2}, \cdots$, can be found on $S$ dividing it into a corresponding sequence of "zones," $Z_{1}, Z_{2}, \ldots$. The first zone $Z_{1}$ is the portion of $S$ containing the vertex and bounded by (but not including) the parallel $P_{1}$. The $n$th zone $Z_{n}$, for $n>1$, is the portion of $S$ bounded by $P_{n-1}$ and $P_{n}$, including the points of the former parallel, but not those of the latter. In the case of a surface whose generating curve is tangent to $O x$ at $O$, it is shown that every point of the $n$th zone is the 1 st, 2 nd, $\cdots,(n-1)$ th double point of certain geodesics of $S$, but is a double point of higher order of no geodesic of $S$. In the case of $Z_{1}$ this is taken to mean that no point of this first zone, or "cap," is the double point of any geodesic of $S$. In the case of a generatrix not tangent to the $x$-axis this conclusion appears in a suitably modified form. The zones may be finite or infinite in number; and in particular the cap may extend over the whole of $S$.

Presented to the Society, February 23, 1946; received by the editors March 28, 1944, and, in revised form, August 14, 1945. 
The results sketched here are developed in the first three sections of the paper.

$\$ 4$ is devoted to a classification of admissible surfaces in accordance with the number of the double points of their geodesics. Either every geodesic of $S$ (the meridians always excepted) has infinitely many double points ("cylindrical surface," or "surface of the first kind"), or else each geodesic has but a finite number of them ("surface of the second kind"). Surfaces of the second kind are in turn of one of two types: either there is an upper bound to the number of double points on any geodesic of $S$ ("conical surface"), or else it is possible to find geodesics the number of whose double points is arbitrarily large ("semi-cylindrical surface"). The existence of this latter class of surfaces seems not to have been specifically remarked before. Appropriate analytic criteria are set up to distinguish between the several classes.

In $\$ 5$ a small class of examples is adduced. While these are somewhat artificial in nature, they serve very well to illustrate the material, and furnish counterexamples to the converses of several of the earlier theorems.

In $\$ 6$ it is indicated briefly that the condition of a continuously turning tangent for the generating curve can in large measure be relaxed without in any way impairing the validity of our conclusions.

The zoning property appears first to have been established for the paraboloid of revolution in a paper by the author and Leon Recht ( $A$ theorem concerning the geodesics on a paraboloid of revolution, Bull. Amer. Math. Soc. vol. 47 (1941)). However, a kind of first zone, or cap, was discussed by CohnVossen for surfaces of a somewhat more general nature (Totalkrïmmung und geodätische Linien auf einfachzusammenhängenden offenen vollständigen Flächen, Rec. Math. (Mat. Sbornik) N.S. vol. 43 (1936)).

1. The nature of the geodesics. Let $S$ be the surface obtained by revolving the curve $y=f(x)$ around the $y$-axis. We shall suppose that:

1. $f(x)$ is continuous and single-valued for $x \geqq 0$.

2. $f(x)$ has everywhere a continuous derivative, including a finite right derivative at $x=0$.

3. $f^{\prime}(x)$ is everywhere positive except possibly at $x=0$, where it may vanish.

As coordinates of a point $P$ (not the vertex) of $S$ we choose $x$, the perpendicular distance of $P$ from the axis of $S$, and $\theta$, the angle which the halfplane determined by $P$ and the axis makes with some fixed half-plane containing the axis. The quantity $x$ is essentially positive, and the sign of $\theta$ is determined by a suitable convention. The coordinate network is thus the orthogonal system composed of the semi-meridians $\theta=$ const. and the parallels $x=$ const.

If we write $\mu(x)=\left(1+f^{\prime 2}(x)\right)^{1 / 2}$, the differential equation of the geodesics of $S$ becomes (cf. Darboux, Théorie des surfaces, vol. 3)

$$
d \theta / d x=t \mu(x) / x\left(x^{2}-t^{2}\right)^{1 / 2},
$$


where $t$ is a parameter which may assume any real value. Corresponding to $t=0$ are the semi-meridians $\theta=$ const. These need concern us no further, so that we may assume $t \neq 0$. The totality of extremals can be written in the form

$$
\theta=\theta_{1}+t \int_{|t|}^{x} \frac{\mu(z) d z}{z\left(z^{2}-t^{2}\right)^{1 / 2}} .
$$

A fixed positive value of $t$ in (1.2) yields one branch of a geodesic, the corresponding negative value the other branch. The two meet at $x=t, \theta=\theta_{1}$, the "point of symmetry" of the geodesic, and the curve is tangent to the parallel of $S$ which passes through this point. It is clear from (1.2) that the two branches of the extremal are symmetric with respect to the plane containing the point of symmetry and the axis of $S$-the "plane of symmetry." Moreover, $\theta$ increases or decreases monotonically with $x$ according as $t$ is positive or negative. Accordingly the geodesics may possess no singularities other than double points, and these must lie in the plane of symmetry.

If we fix $|t|,(1.2)$ represents a one-parameter family of geodesics, each tangent at its point of symmetry to the parallel $x=|t|$. These curves are all congruent, so that we may single out for study any representative member, say that for which $\theta_{1}=0$, and call it the "geodesic $(t)$. " Moreover, owing to the symmetry of the curve, we may confine our attention to that branch for which $t>0$. We have thus as the object of our investigation the equation

$$
\theta=t \int_{t}^{x} \frac{\mu(z) d z}{z\left(z^{2}-t^{2}\right)^{1 / 2}} \quad(x \geqq t>0) .
$$

Roughly speaking, $\theta$ is the amount by which the geodesic $(t)$ winds around the axis of $S$ between its point of symmetry and its intersection with the parallel $x$.

2. The functions $\theta(x, t)$ and $\theta^{*}(x)$. We introduce a function $\theta(x, t)$. whose domain of definition is the sector of the $(x, t)$-plane bounded by the lines $t=0$ and $t=x$, and with the origin deleted. It is defined as follows:

$$
\begin{aligned}
& \theta(x, t)=t \int_{t}^{x} \frac{\mu(z) d z}{z\left(z^{2}-t^{2}\right)^{1 / 2}} \\
& \theta(x, x)=0 \\
& \theta(x, 0)=\mu(0) \pi / 2
\end{aligned}
$$$$
\text { for } x>0 \text { and } 0<t<x \text {, }
$$$$
\text { for } x>0 \text {, }
$$$$
\text { for } x>0 \text {. }
$$

LEMMA 2.1. $\theta(x, t)$ is continuous throughout its domain. form

This is most readily seen if we cast the integral of the definition in the

$$
\theta(x, t)=\int_{1}^{x / t} \frac{\mu(w t) d w}{w\left(w^{2}-1\right)^{1 / 2}}
$$

by means of the substitution $z=w t$. It is evident on the basis of well known 
theorems concerning generalized integrals that $\theta(x, t)$ is continuous, as defined, for $0<t<x$ and for $x=t(\neq 0)$. The proof of its continuity for $t=0(x \neq 0)$ can be sketched as follows:

Choose any $x_{1}>0$, and an as yet unspecified positive number $\epsilon$. Let $\delta t$ be less than $\epsilon$, and write

$$
\theta\left(x_{1}, \delta t\right)=\int_{1}^{\epsilon / \delta t}+\int_{\epsilon / \delta t}^{x_{1} / \delta t} \frac{\mu(w \delta t) d w}{w\left(w w^{2}-1\right)^{1 / 2}}=I+J .
$$

Denoting by $A$ the upper bound of $\mu(x)$ in $0 \leqq x \leqq x_{1}$, then we have $0 \leqq J$ $\leqq A\left(\operatorname{arcsec}\left(x_{1} / \delta t\right)-\operatorname{arcsec}(\epsilon / \delta t)\right)$. No matter what fixed value is given to $\epsilon, \delta t$ can be taken so small as to make the right member of this inequality vanishingly small. In the integral $I$, the argument of $\mu$ is confined to the interval $\delta t \leqq x \leqq \epsilon$, and, for some $\zeta$ between $\delta t$ and $\epsilon, I=\mu(\zeta) \operatorname{arcsec} \epsilon / \delta t$. One can now adjust $\epsilon$ so that $\mu(\zeta)$, which is continuous at the origin, differs by an arbitrarily small amount from $\mu(0)$, and then take $\delta t$ so small that $\operatorname{arcsec}(\epsilon / \delta t)$ approximates as closely as is desired to $\pi / 2$. Thus, for any $x_{1}>0, \theta\left(x_{1}, \delta t\right)$ approaches $\mu(0) \pi / 2$ as $\delta t$ tends to zero. To complete the proof it is merely necessary to remark that $\left\{\theta\left(x_{1}+\delta x, \delta t\right)-\theta\left(x_{1}, 0\right)\right\}=\left\{\theta\left(x_{1}+\delta x, \delta t\right)-\theta\left(x_{1}, \delta t\right)\right\}$ $+\left\{\theta\left(x_{1}, \delta t\right)-\theta\left(x_{1}, 0\right)\right\}$, and to observe that for any $\delta t>0$ the first expression in braces on the right-hand side tends to zero with $\delta x$, in accordance with known theorems on integrals.

For a fixed positive value of $x, \theta(x, t)$ is a continuous function of $t$ in the interval $0 \leqq t \leqq x$, and consequently must attain its upper bound at some point (possibly of course at several points) of this interval. The value of this upper bound we denote by $\theta^{*}(x)$, and we set as a matter of definition $\theta^{*}(0)=\mu(0) \pi / 2$. The geometric significance of this new function can be brought out in the following way. Let $r(Q)$ denote the line segment dropped from the point $Q$ of $S$ normal to the axis of $S$. As $Q$ traces the geodesic $(t)$ from its point of symmetry to its intersection with parallel $x, r(Q)$ undergoes a rotation $\theta(x, t)$. For at least one geodesic $(t)$ the amount of this rotation must be an absolute maximum; and this maximal rotation is $\theta^{*}(x)$.

\section{LEMMA 2.2. $\theta^{*}(x)$ is continuous and nondecreasing for $0 \leqq x<\infty$.}

It is apparent from the defining equations (2.1) that, for a given $t, \theta(x, t)$ increases or remains constant as $x$ increases. Consequently its upper bound in $0 \leqq t \leqq x$ cannot decrease when $x$ increases. That $\lim _{x \rightarrow 0} \theta^{*}(x)=\mu(0) \pi / 2$ was implied in the proof of the preceding lemma. The proof that $\theta^{*}(x)$ is continuous at the interior points of its domain is a perfectly straightforward one, and need not be given here.

As $x$ becomes infinite $\theta^{*}(x)$ may itself become infinite, or it may approach a finite limit. The discussion of the conditions which govern these important alternatives will be reserved for a later section, in order not to interrupt the continuity of the present line of investigation. 
3. The zones. Let $Q$ be a double point of the geodesic $(t)$. That portion of the complete geodesic lying between the parallel through $Q$ and the parallel through its point of symmetry $P$ we call a "geodesic loop." $Q$ is the "vertex" of the loop, and by its "order" we mean the total number of its double points, $Q$ included. Since $P$ and $Q$ lie in the plane of symmetry, their angular coordinates, which are $O$ and $\theta(x, t)$ respectively, differ by a multiple of $\pi$. This multiple is precisely the order of the loop, since each increase of $\theta(x, t)$ by the value $\pi$ marks another intersection of the plane of symmetry by the two branches of the geodesic, that is, another double point. We have established

Lemma 3.1. A necessary and sufficient condition that the vertex of a geodesic loop of order $k$ lie on the parallel $x$ is that, for some $t, 0<t<x$, we shall have $\theta(x, t)=k \pi$.

$\theta(x, t)$ as a continuous function of $t$ must assume in the interval $0 \leqq t \leqq x$ every value between its lower bound zero (for $t=x$ ) and its upper bound $\theta^{*}(x)$. From this follows

LEMMA 3.2. If $k$ is a positive integer or zero, and if $k \pi \leqq \theta^{*}(x)<(k+1) \pi$, then every point of the parallel $x$ is the vertex of at least one loop of each of the orders $1,2, \cdots, k$, but is the vertex of no loop of higher order.

Let $N$ denote the smallest integer greater than $\mu(0) / 2 . N$ must be at least equal to unity, for $\mu(x) \geqq 1$. By definition $\theta^{*}(0)=\mu(0) \pi / 2$, and accordingly

$$
(N-1) \pi \leqq \theta^{*}(0)<N \pi \text {. }
$$

Since $\theta^{*}(x)$ increases continuously, there exists a strictly increasing sequence of positive values of $x, \xi_{1}, \xi_{2}, \cdots$, each $\xi_{n}$ being the smallest number with the property

$$
\theta^{*}\left(\xi_{n}\right)=(N+n-1) \pi \quad(n=1,2,3, \cdots) .
$$

This sequence is infinite if $\lim _{x \rightarrow \infty} \theta^{*}(x)=\infty$. If, on the other hand, $\lim _{x \rightarrow \infty} \theta^{*}(x)$ $=\lambda<\infty$, the sequence ends with the term $\xi_{M-N+1}, M$ being the integer defined by the inequalities $\pi M<\lambda \leqq \pi(M+1)$.

The sequence $\left(\xi_{n}\right)$ determines a sequence of parallels $x=\xi_{1}, x=\xi_{2}, \cdots$, which divide $S$ into "zones." The first zone is characterized by the inequality $0 \leqq x<\xi_{1}$, the $n$th zone $(n>1)$ by $\xi_{n-1} \leqq x<\xi_{n}$. It follows from (3.1), (3.2) and the monotonicity of $\theta^{*}(x)$ that, for every point of the $n$th zone $(n=1,2,3, \cdots)$, $(N+n-2) \pi \leqq \theta^{*}(x)<(N+n-1) \pi$. An application of Lemma 3.2 then yields

THEOREM 1. There exists a sequence of parallels $x=\xi_{1}, x=\xi_{2}, \cdots$ dividing $S$ into zones, as described above. Every point of the nth zone is the vertex of at least one loop of each of the orders $1,2, \cdots, N+n-2$ (where $N$ is the smallest integer greater than $\mu(0) / 2)$, but is the vertex of no loop of higher order.

In the important case for which $f^{\prime}(0)=0$, we have $\mu(0)=1$, and likewise 
$N=1$. The theorem just proved indicates that no point of the first zonewhich in this case we call the "cap" of $S$-can be a double point of any geodesic of $S$.

4. The number of windings and the index. Surfaces of the sort under discussion fall into two classes. On a "surface of the first kind" every geodesic winds an infinite number of times around the axis, while on a "surface of the second kind" each geodesic makes but a finite number of turns. No intermediate case is possible. Surfaces of the first kind we shall sometimes call "cylindrical," since with respect to the number of windings the geodesics of such a surface behave as do those on a circular cylinder.

Illustrative of these surfaces of the first kind is the paraboloid of revolution. As an example of a surface of the second kind we may cite the cone, to be specific let us say the cone generated by revolving the line $y=m x$ about $O y$. It is then a simple matter to show that $\left(1+m^{2}\right)^{1 / 2} / 4$ furnishes an upper bound for the number of turns which the geodesics of $S$ may make about its axis. Surfaces of the second kind having the property that the number of windings of their geodesics possesses an upper bound we accordingly call "conical." On the other hand, it is an interesting fact that there exist admissible surfaces of the second kind for which no such bound exists, that is, on which one can find geodesics the number of whose windings is arbitrarily large. Such surfaces we call "semi-cylindrical," since, in common with the cylindrical surfaces, they possess infinitely many zones.

In this section we propose to demonstrate these facts and to set up various analytic criteria.

THEOREM 2. $S$ is of the first or second kind according as

$$
J=\int^{\infty} \frac{\mu(z)}{z^{2}} d z
$$

diverges or converges.

For $J$ converges or diverges with

$$
t \int_{t}^{\infty} \frac{\mu(z) d z}{z\left(z^{2}-t^{2}\right)^{1 / 2}}
$$

and this integral represents the total number of windings of the geodesic $(t)$, that is, the limit of $\theta(x, t)$ as $x$ approaches infinity.

We assume henceforth that $J$ exists, and define the "index" $\phi(t)$ of the geodesic $(t)$ as follows:

$$
\begin{array}{cc}
\phi(t)=t \int_{t}^{\infty} \mu(z) d z / z\left(z^{2}-t^{2}\right)^{1 / 2} & \text { for } t>0, \\
\phi(0)=\mu(0) \pi / 2 . &
\end{array}
$$

LEMma 4.1. $\phi(t)$ is continuous for $0 \leqq t<\infty$. 
That $\phi(t)$ is continuous for $t>0$ becomes clear when the integral is written in the form

$$
\int_{1}^{\infty} \frac{\mu(w t) d w}{w\left(w^{2}-1\right)^{1 / 2}}
$$

by means of the substitution $z=w t$. To demonstrate its continuity at $t=0$, we write

$$
\begin{gathered}
\phi(t)=t \int_{t}^{1}+t \int_{1}^{\infty} \frac{\mu(z) d z}{z\left(z^{2}-t^{2}\right)^{1 / 2}}, \\
\phi(t)-t \int_{t}^{1} \frac{\mu(z) d z}{z\left(z^{2}-t^{2}\right)^{1 / 2}}<t \int_{1}^{\infty} \frac{\mu(z) d z}{z\left(z^{2}-1\right)^{1 / 2}} .
\end{gathered}
$$

The second of the latter pair of terms tends to zero with $t$, while the former tends to $\mu(0) \pi / 2$, as follows from Lemma 2.1.

The index $\phi(t)$ represents the total angle of turn made by either branch of the geodesic $(t)$ around the axis of $S$. Thus a surface is conical or cylindrical according as its index function is bounded or unbounded.

In the discussion of the conditions which determine the boundedness or the unboundedness of $\phi(t)$, four functions (two of them very closely related) play a prominent role. They are

$$
\begin{array}{ll}
\alpha(t)=\frac{1}{t^{1 / 2}} \int_{0}^{t 1 / 2} \mu\left(z^{2}+t\right) d z & (t>0 ; \alpha(0)=\mu(0)), \\
\beta(t)=t \int_{t}^{\infty} \frac{\mu(z)}{z^{2}} d z & (t>0 ; \beta(0)=\mu(0)), \\
\gamma(t)=\frac{1}{t} \int_{t}^{2 t} \mu(z) d z & (t>0 ; \gamma(0)=\mu(0)), \\
\delta(t)=\frac{1}{t^{1 / 2}} \int_{t}^{2 t} \frac{\mu(z) d z}{(z-t)^{1 / 2}} & (t>0 ; \delta(0)=2 \mu(0)) .
\end{array}
$$

LEMMA 4.2. The functions $\alpha(t), \beta(t), \gamma(t)$, and $\delta(t)$ are continuous for $0 \leqq t<\infty$, and

$$
\delta(t)=2 \alpha(t) .
$$

The latter part of the lemma is proved by replacing $z$ by $z^{2}+t$ in the integral for $\delta(t)$. The continuity of the four functions for $t>0$ is evident, and we need only show that $\alpha(t), \beta(t)$, and $\gamma(t)$ approach $\mu(0)$ as $t$ tends to zero. If we observe that

$$
\alpha(t)=\frac{1}{t^{1 / 2}} \mu\left(\tau^{2}+t\right) \int_{0}^{t / 2} d z=\mu\left(\tau^{2}+t\right), \quad 0<\tau<t^{1 / 2},
$$

the desired result follows upon letting $t$ approach zero. A similar proof applies 
for $\gamma(t)$. To take care of $\beta(t)$, we choose a positive number $\epsilon$, and write

$$
\begin{aligned}
& \beta(t)=t \int_{t}^{\cdot}+t \int_{0}^{\infty} \frac{\mu(z)}{z^{2}} d z=t \mu(\tau) \int_{t}^{e} \frac{d z}{z^{2}}+t \int_{0}^{\infty} \frac{\mu(z)}{z^{2}} d z \quad(t<\tau<\epsilon), \\
& \beta(t)-\mu(0)=\mu(\tau)-\mu(0)-\frac{t}{\epsilon} \mu(\tau)+t \int_{0}^{\infty} \frac{\mu(z)}{z^{2}} d z .
\end{aligned}
$$

Because of continuity considerations we may take $\epsilon$ so that $\mu(\tau)-\mu(0)$ is arbitrarily small for any $\tau<\epsilon$. Allowing $t$ to tend to zero, the last pair of terms become vanishingly small, and we conclude $\beta(t) \rightarrow \mu(0)$.

LEMMA 4.3. There exist positive constants $c_{1}, c_{2}, c_{8}$, and $c_{4}$ such that, for $t \geqq 0$,

$$
c_{1} \alpha(t)+c_{2} \beta(2 t) \leqq \phi(t) \leqq c_{3} \alpha(t)+c_{4} \beta(2 t) .
$$

We may write $\phi(t)=I_{1}+I_{2}$,

$$
I_{1}=t \int_{t}^{2 t} \frac{\mu(z) d z}{z\left(z^{2}-t^{2}\right)^{1 / 2}}, \quad I_{2}=t \int_{2 t}^{\infty} \frac{\mu(z) d z}{z\left(z^{2}-t^{2}\right)^{1 / 2}} .
$$

In the interval $t \leqq x \leqq 2 t(t>0)$

$$
\frac{1}{2 t(3 t)^{1 / 2}} \leqq \frac{1}{x(x+t)^{1 / 2}} \leqq \frac{1}{t(2 t)^{1 / 2}}
$$

and consequently

$$
\frac{1}{2(3)^{1 / 2}} \frac{1}{t^{1 / 2}} \int_{t}^{2 t} \frac{\mu(z) d z}{(z-t)^{1 / 2}} \leqq I_{1} \leqq \frac{1}{2^{1 / 2}} \frac{1}{t^{1 / 2}} \int_{t}^{2 t} \frac{\mu(z) d z}{(z-t)^{1 / 2}}
$$

or, making use of (4.3d) and Lemma 4.2,

$$
\alpha(t) / 3^{1 / 2} \leqq I_{1} \leqq 2^{1 / 2} \alpha(t) .
$$

For the second integral we have at once

$$
I_{2} \geqq t \int_{2 t}^{\infty} \frac{\mu(z) d z}{z^{2}}=\frac{1}{2} \beta(2 t) .
$$

On the other hand, we may write

$$
I_{2}=t \int_{2 t}^{\infty} \frac{\mu(z)}{z^{2}} \frac{z}{\left(z^{2}-t^{2}\right)^{1 / 2}} d z .
$$

The second factor of the integrand is a monotonically decreasing function of $z$ in $2 t \leqq z<\infty$, and is bounded in that interval by $2 / 3^{1 / 2}$. Thus

$$
I_{2} \leqq \frac{2}{3^{1 / 2}} t \int_{2 t}^{\infty} \frac{\mu(z)}{z^{2}} d z=\frac{1}{3^{1 / 2}} \beta(2 t)
$$


Combining (4.6), (4.7a), and (4.7b) we obtain

$$
\alpha(t) / 3^{1 / 2}+\beta(2 t) / 2 \leqq \phi(t) \leqq 2^{1 / 2} \alpha(t)+\beta(2 t) / 3^{1 / 2} .
$$

A simple numerical calculation shows that these inequalities subsist for $t=0$. An immediate consequence of Lemma 4.3 is

Lemma 4.4. $A$ necessary and sufficient condition that $\phi(t)$ be bounded in $0 \leqq t<\infty$ is that $\alpha(t)$ and $\beta(t)$ be bounded in the same interval.

LEMMA 4.5. The boundedness of $\alpha(t)$ in $0 \leqq t<\infty$ implies that of $\beta(t)$.

Let us suppose that for $t \geqq 0$ we have $\alpha \leqq A / 2$, so that, by Lemma 4.2, $\delta(t) \leqq A$. Now it is clear that

$$
\begin{aligned}
\delta(t) & =\frac{1}{t^{1 / 2}} \int_{t}^{2 t} \frac{\mu(z) d z}{(z-t)^{1 / 2}} \geqq \frac{1}{t} \int_{t}^{2 t} \mu(z) d z \\
& =t \int_{t}^{2 t} \frac{\mu(z)}{t^{2}} d z \geqq t \int_{t}^{2 t} \frac{\mu(z)}{z^{2}} d z
\end{aligned}
$$

and thus, for all $t \geqq 0$,

$$
t \int_{t}^{2 t} \frac{\mu(z)}{z^{2}} d z \leqq \delta(t) \leqq A .
$$

This inequality continues to hold if we replace $t$ by $2^{n} t(n=1,2, \cdots)$ :

$$
t \int_{2 n t}^{2^{n+1} t} \frac{\mu(z)}{z^{2}} d z \leqq \frac{1}{2^{n}} A .
$$

Adding corresponding sides for $n=1,2, \cdots, k$ gives

$$
t \int_{t}^{2 k+1 t} \frac{\mu(z)}{z^{2}} d z \leqq A\left(1+\frac{1}{2}+\cdots+\frac{1}{2^{k}}\right)<2 A
$$

and, upon letting $k$ increase without limit, we find that $\beta(t) \leqq 2 A$.

THEOREM 3. A surface of the second kind is conical or semi-cylindrical according as $\alpha(t)$ (or $\delta(t)$ ) is or is not bounded as $t \rightarrow \infty$.

This is a direct consequence of Lemmas 4.2, 4.4, and 4.5.

THEOREM 4. A necessary condition that a surface of the second kind be conical is that

be bounded as $t \rightarrow \infty$.

$$
\gamma(t)=\frac{1}{t} \int_{t}^{2 t} \mu(z) d z
$$

For the first inequality of (4.8) shows that $\delta(t) \geqq \gamma(t)$, and the rest follows from Theorem 3. 
In practice this test is frequently decisive, and it has the advantage of being easily applied. It should be remarked how severely it restricts the class of functions $\mu(x)$ which give rise to conical surfaces, for it implies that the mean value of $\mu(x)$ over the interval $t \leqq x \leqq 2 t$ must be bounded as $t \rightarrow \infty$. Even though $\mu$ itself may be unbounded, it must still possess in this sense the asymptotic quality of a constant.

From Theorems 3 and 4 follow

THEOREM 5A. The surface $S$ is conical if $\mu(x)$ is bounded.

Theorem 5B. A surface of the second kind is semi-cylindrical if $\lim \inf _{x \rightarrow \infty} \mu(x)=\infty$.

In the case of the important subclass of admissible surfaces for which $f^{\prime}(x)$-and consequently $\mu(x)$-increases monotonically, the criteria become particularly simple:

TheOREM 6A. A surface of monotonically increasing $\mu(x)$ is:

(a) conical if $\mu(x)$ is bounded;

(b) semi-cylindrical if $J$ exists and $\mu(x) \rightarrow \infty$;

(c) cylindrical if $J$ fails to exist.

These are merely restatements of Theorems $5 \mathrm{~A}, 5 \mathrm{~B}$, and 2 respectively Somewhat more generally we have the following theorem.

THEOREM 6B. If for $x$ large $\mu(x) \sim x^{\alpha}$, then $S$ is:

(a) cylindrical for $\alpha \geqq 1$;

(b) semi-cylindrical for $0<\alpha<1$;

(c) conical for $\alpha<0$.

5. Illustrative examples. Surfaces of monotonically increasing $\mu(x)$ are easily exemplified by virtue of Theorem 6 . In this section we shall introduce a special class of surfaces of non-monotonic $\mu(x)$, which serve very well to illuminate much of the foregoing material.

Let $h_{n}$ and $d_{n}(n=1,2,3, \cdots)$ be positive numbers subject for the pressent only to the conditions $d_{n} 2^{-2 n} \rightarrow 0\left(d_{n}<2^{2 n}\right)$ and $h_{n} \rightarrow \infty$. We take for $\mu(x)$ the continuous function whose ordinate maintains the constant value 2 except over the intervals $2^{2 n} \leqq x \leqq 2^{2 n}+2 d_{n}(n=1,2,3, \cdots)$. Over the first half of the $n$th of these exceptional intervals it rises linearly from 2 to $2+h_{n}$, while over the second half it drops linearly back to 2 . Analytically

(5.1a) $\mu(x)=2+\left(h_{n} / d_{n}\right)\left(x-2^{2 n}\right)$

(5.1c) $\mu(x)=2$

$$
\begin{aligned}
\text { for } \quad 2^{2 n} \leqq x \leqq & 2^{2 n}+d_{n}, \\
\text { for } 2^{2 n}+d_{n} \leqq x \leqq & 2^{2 n}+2 d_{n}, \\
& \text { elsewhere. }
\end{aligned}
$$

The generating curve itself may be described as a sequence of line segments 
of slope $3^{1 / 2}$ connected by $S$-shaped arcs whose central portions become increasingly steep. This surface we shall call $S\left(h_{n}, d_{n}\right)$.

THEOREM 7A. The surface $S\left(h_{n}, d_{n}\right)$ is of the first kind if the quantities $h_{n} d_{n} 2^{-4 n}$ are bounded away from zero.

Taking the lower limit of the integral $J$ as unity, we have

$$
J=\int_{1}^{\infty} \frac{\mu(z)}{z^{2}} d z=\int_{1}^{\infty} \frac{2}{z^{2}} d z+\lim _{N \rightarrow \infty} \sum_{n=1}^{N} \int_{2^{2 n}}^{2^{2 n}+2 d_{n}} \frac{\mu(z)-2}{z^{2}} d z
$$

Denoting the sum in the final term by $s_{N}$, we obtain the following inequality by integrating over only the first half of each of the exceptional intervals. (We remark that $\mu(z)-2$ is different from zero only over these intervals.)

$$
s_{N}>\sum_{1}^{N} \int_{2^{2 n}}^{2^{2 n}+d_{n}} \frac{h_{n}}{d_{n}} \frac{z-2^{2 n}}{z^{2}} d z=\sum_{1}^{N} \frac{h_{n}}{d_{n}}\left\{\log \left(1+\frac{d_{n}}{2^{2 n}}\right)-\frac{d_{n}}{2^{2 n}+d_{n}}\right\} .
$$

Now for $x>0, \log (1+x)>x-x^{2} / 2$, and

$$
s_{N}>\sum_{1}^{N} \frac{h_{n} d_{n}}{2^{4 n}}\left\{\frac{1}{1+d_{n} 2^{-2 n}}-\frac{1}{2}\right\} \text {. }
$$

Since by hypothesis $d_{n} 2^{-2 n} \rightarrow 0$, the factor in braces of this last expression is greater say than $1 / 4$ for $n$ large. If, then, the sequence $h_{n} d_{n} 2^{-4 n}$ is bounded away from zero, $\lim _{N \rightarrow \infty} s_{N}=\infty$, and $J$ fails to exist.

THEOREM 7B. The surface $S\left(h_{n}, d_{n}\right)$ is of the second kind if the quantities $h_{n} d_{n} 2^{-8 n}$ are bounded.

Using the representation (5.2), it is easily verified that

$$
s_{N}<\sum_{1}^{N} \int_{2^{2 n}}^{2^{2 n}+2 d_{n}} \frac{h_{n}}{z^{2}} d z<2 \sum_{1}^{N} \frac{h_{n} d_{n}}{2^{4 n}} .
$$

This latter sum clearly has a limit as $N \rightarrow \infty$ provided the condition of the hypothesis holds, and thus $J$ exists.

THEOREM 8A. The surface $S\left(h_{n}, d_{n}\right)$ is conical if the quantities $h_{n}{ }^{2} d_{n} 2^{-2 n}$ are bounded.

Since, as we assumed at the outset, $\lim _{n \rightarrow \infty} h_{n}=\infty$, the condition of the hypothesis implies that the sequence $h_{n} d_{n} 2^{-3 n}$ is bounded, so that by the preceding theorem $J$ exists. We shall now show that $\delta(t)$ is bounded. First it should be remarked that, for any value of $t$, the range of integration of

$$
\delta(t)=\frac{1}{t^{1 / 2}} \int_{t}^{2 t} \frac{\mu(z) d z}{(z-t)^{1 / 2}}
$$


contains points of at most one of the exceptional intervals. If no point of such an interval occurs in the range, $\mu(x)$ maintains the constant value 2 , and $\delta(t)=4$. In the contrary case we may suppose that the range contains the $n$th interval in its entirety. It can be verified that this assumption involves no loss of generality. Then, since $t>2^{2(n-1)}$,

$$
\begin{aligned}
\delta(t) & =4+\frac{1}{t^{1 / 2}} \int_{2^{2 n}}^{2^{2 n}+2 d_{n}} \frac{(\mu(z)-2)}{(z-t)^{1 / 2}} d z \\
& <4+\frac{2}{2^{n}} \int_{2^{2 n}}^{2^{2 n}+2 d_{n}} \frac{\mu(z)-2}{(z-t)^{1 / 2}} d z .
\end{aligned}
$$

On the other hand, the integral over the first half of its range is greater than that over the second half, for at the two points of equal $\mu$ the denominator in the first half is less than in the second. Consequently

$$
\delta(t)<4+\frac{4}{2^{n}} \int_{2^{2 n}}^{2^{2 n+d_{n}}} \frac{z-2^{2 n}}{(z-t)^{1 / 2}} d z .
$$

By direct evaluation of this integral we find

$$
\delta(t)<4+\frac{8}{3} \frac{h_{n}\left(d_{n}\right)^{1 / 2}}{2^{n}} .
$$

Thus, under the given conditions, $\delta(t)$ is bounded and $S$ conical (Theorem 3B).

COROLLARY. The converse of Theorem 5A fails to hold.

For the condition of Theorem $8 \mathrm{~A}$ can be met (in a multiplicity of ways) with unbounded $h_{n}$ 's, that is, with $\mu(x)$ unbounded.

THEOREM 8B. The surface $S\left(h_{n}, d_{n}\right)$ is semi-cylindrical if the sequence $h_{n} d_{n} 2^{-3 n}$ is bounded, but $h_{n}{ }^{2} d_{n} 2^{-2 n}$ is not.

Here $J$ exists, and the surface is of the second kind. Starting with (5.3), a laborious calculation, not reproduced here, yields

$$
\delta\left(2^{2 n}\right)=A+B h_{n}\left(d_{n}\right)^{1 / 2} 2^{-n}
$$

where $A$ and $B$ are constants independent of $n$. Thus, under the given conditions, $\delta(t)$ is unbounded, and the surface semi-cylindrical (Theorem $3 \mathrm{~B}$ ).

This furnishes us with an example of a surface of semi-cylindrical type for which $\lim \inf _{x \rightarrow \infty} \mu(x)$ is not infinite.

THEOREM 9. The converse of Lemma 4.5 fails to hold.

To show this, let $S$ be such that the sequence $h_{n} d_{n} 2^{-2 n}$ (and consequently $h_{n} d_{n} 2^{-8 n}$ ) is bounded, while $h_{n}^{2} d_{n} 2^{-2 n}$ is not. By Theorem $8 \mathrm{~B}, S$ is semi-cylindri- 
cal, and $\alpha(t)$ is unbounded (Theorem 3A). Now it is not difficult to show that, under the given conditions, $\beta(t)$ is bounded provided the quantities $\beta\left(2^{2 n}\right)$ are bounded. Over the $n$th exceptional interval $\mu(x) \leqq 2+h_{n}$, and

$$
\begin{aligned}
\beta\left(2^{2 n}\right) & \leqq 2^{2 n} \int_{2^{2 n}}^{\infty} \frac{2 d z}{z^{2}}+\sum_{k=0}^{\infty} 2^{2 n} \int_{2^{2(n+k)}}^{2^{2(n+k)}+2 d_{n+k}} \frac{h_{n+k}}{z^{2}} d z \\
& =2+2^{2 n} \sum_{k=0}^{\infty} h_{n+k}\left\{\frac{1}{2^{2(n+k)}}-\frac{1}{2^{2(n+k)}+2 d_{n+k}}\right\} \\
& =2+2 \sum_{k=0}^{\infty} \frac{h_{n+k} d_{n+k}}{2^{2(n+k)}} \frac{1}{2^{2 k}+2 d_{n+k} 2^{-2 n}} \\
& <2+2 \sum_{k=0}^{\infty} \frac{h_{n+k} d_{n+k}}{2^{2(n+k)}} \frac{1}{2^{2 k}} .
\end{aligned}
$$

Given, then, that $h_{n} d_{n} 2^{-2 n}$ is less than some positive $A_{\mathrm{a}}^{\text {for }}$ all $n$, we conclude that

$$
\beta\left(2^{2 n}\right) \leqq 2+2 A \sum_{0}^{\infty} \frac{1}{2^{2 k}}=2+\frac{8}{3} A .
$$

Thus $\beta(t)$ is bounded, while $\alpha(t)$ is not.

6. The case of discontinuous $f^{\prime}(x)$. For the sake of simplicity, we have assumed up to the present that $f^{\prime}(x)$ is everywhere continuous. In this section we shall show that this restriction can to a large extent be relaxed without invalidating the results of $\S \S 1$ to 4 . We replace the conditions of $\S 1$ by the following:

1. $f(x)$ is continuous and single-valued for $x \geqq 0$.

2. $f^{\prime}(x)$ is continuous except on a discrete sequence of points $x=a_{1}, x=a_{2}$, with $0<a_{1}<a_{2}<\cdots$. At each of these points the left and right derivatives exist, but are different.

3. $f^{\prime}(x)$ is everywhere positive except possibly at $x=0$, where it may vanish.

On $S$, the surface formed by revolving $y=f(x)$ about $O y$, the parallels $x=a_{1}, x=a_{2}, \cdots$ are composed of singular points. These are the "parallels of division," and the regions between consecutive parallels of division we shall call "regions of regularity." The function $\mu(x)$ is continuous throughout any region of regularity, but changes abruptly in value when a parallel of division is crossed. With this in mind, we can again define the "geodesic $(t)$ " as the union of the two branches (1.2) with parameters $t$ equal in magnitude but opposite in sign. When we do so, all the remarks of $\S 1$ remain valid.

If $P_{1}$ and $P_{2}$ are nearby points of the same region of regularity, then it is well known that the arc of the geodesic $(t)$ joining them furnishes the shortest path between them. This is, however, by no means so obvious when these 
points are separated by a parallel of division. The following lemma demonstrates that this is, in fact, the case.

Lemma 6.1. Let $P_{1}$ and $P_{2}$ be nearby points on opposite sides of a parallel of division, and let $\left[P_{1} P_{2}\right]$ denote the geodesic arc, as defined above, which joins them. Then $\left[P_{1} P_{2}\right]$ is the shortest path joining them.

The proof will merely be sketched. It is at once evident that those portions of the shortest path from $P_{1}$ to $P_{2}$ which lie in the two separate regions of regularity must be geodesic arcs. It is thus a question of showing that the minimal path is obtained by preserving the value of the parameter $t$ across the parallel of division.

Let $P_{0}$ be an arbitrary point of the parallel of division, determined by some parameter, say $\theta_{0}$. The parameters $t_{1}$ and $t_{2}$ of the geodesic arcs $\left[P_{1} P_{0}\right]$ and $\left[P_{0} P_{2}\right]$ are functions of $\theta_{0}$. From the differential element of $S, d s^{2}=\mu^{2} d x^{2}$ $+x^{2} d \theta^{2}$, we find that the differential of arc along a geodesic of $S$ is given by

$$
d s=\frac{x \mu(x)}{\left(x^{2}-t^{2}\right)^{1 / 2}} d x .
$$

This enables us to set up the expression $L$, the sum of the arc lengths $\left[P_{1} P_{0}\right]$ and $\left[P_{0} P_{2}\right]$. Differentiating this expression with respect to $\theta_{0}$ and setting the derivative equal to zero, we find that $L$ has an extremum only for $t_{1}=t_{2}$. On geometrical grounds, this must be a minimum of $L$.

Passing to the results of $\$ 2$, the definition and the continuity of $\theta(x, t)$ are handled exactly as in the simpler case. Lemma 2.1 requires that $\mu(x)$ be continuous near $x=0$; but this remains true since we have postulated $a_{1}>0$. For the rest, it is only necessary that $\mu(x)$ be positive, bounded and integrable. Once more $\theta^{*}(x)$ appears as a continuous nondecreasing function of $x$, and the zoning phenomenon reemerges with the results of $\$ 3$ unchanged.

Likewise, the conclusions of $\$ 4$ carry through as before, for the continuity of $\mu(x)$ is required only at the origin.

Thus the whole theory exposed in this paper remains true for the more general type of surfaces considered in this section.

A final brief remark is in order concerning surfaces for which $f^{\prime}(x)$ has an infinite discontinuity. This can come about in one of two ways. The generating curve may have a vertical asymptote for some finite $x$, in which case $S$ is obviously cylindrical. Otherwise, the slope at the origin may be infinite. If $\mu(x)$ increases monotonically to infinity as $x$ decreases to zero, there exists, for any arbitrarily large positive number $A$, a value $\xi$ of $x$ so small that, for $x<\xi, \mu(x)>A$. Then, for $x<\xi$ and $t<x$, we have

$$
\theta(x, t)=t \int_{t}^{x} \frac{\mu(z) d z}{z\left(z^{2}-t^{2}\right)^{1 / 2}}>A t \int_{t}^{x} \frac{d z}{z\left(z^{2}-t^{2}\right)^{1 / 2}}=A \operatorname{arcsec} \frac{x}{t} .
$$


By choosing $t$ sufficiently small, we can make $\theta(x, t)>A$. As a moving point $P$ traces the geodesic $(t)$ from the point $x$ to its point of symmetry, the radius vector $r(P)$ rotates around the axis of $S$, and the amount of this rotation can be made arbitrarily large by taking $t$ small enough. It is obvious that here the zoning property disappears entirely.

Columbia University, NEW YORK, N. Y. 